\title{
Virulence comparisons of high-temperature-adapted Heterorhabditis bacteriophora, Steinernema feltiae and S. carpocapsae
}

\author{
I. A. SUSURLUK, T. C. ULU \\ Uludag University, Agriculture Faculty, Plant Protection Department, 16059 Nilüfer, Bursa, Turkey, E-mail: susurluk@uludag.edu.tr
}

\section{Article info}

Received October 14, 2014 Accepted November 24, 2014

\begin{abstract}
Summary
Entomopathogenic nematodes (EPNs) are environmentally safe alternative control agents. Nematodes in the Heterorhabditidae and Steinernematidae families are widely used in biological control frameworks, especially for soil-inhabiting insect pests. In this experiment, Heterorhabditis bacteriophora (Poinar, 1976), Steinernema feltiae (Filipjev, 1934) and S. carpocapsae (Weiser, 1955) adapted at high temperature were assessed in order to detect differences in virulence between adapted and non-adapted populations. All species were exposed to $38^{\circ} \mathrm{C}$ for $2 \mathrm{~h}$. After this treatment, live infective juveniles (IJs) were used to infect to last instar Galleria mellonella (Linnaeus, 1758). larvae at the following doses: $1,2,3,4$ and $5 \mathrm{IJ}$ /larva. The $\mathrm{LD}_{50}$ and $\mathrm{LD}_{90}$ were obtained for these species. Non-adapted populations of the nematode species were used as controls for this experiment. The results indicated that differences in $S$. feltiae and $S$. carpocapsae virulence between the adapted and non-adapted populations were significant; no significant difference was observed between the adapted and non-adapted $H$. bacteriophora populations.
\end{abstract}

Keywords: Heterorhabditis bacteriophora; Steinernema feltiae; S. carpocapsae; temperature; adaptation; virulence

\section{Introduction}

Due to the hazardous effects of agricultural chemicals on humans and the environment, pesticide resistance and low MRL (Maximum Residue Limits) policies on agricultural products, biological control has become one of the most alternative control methods. In the biological control framework, entomopathogenic nematodes (EPNs) from the Steinernematidae and Heterorhabditidae families have been used to control soil-dwelling insect pests for a few decades, especially in Europe and the USA (Shapiro \& Gaugler, 2010). EPNs are safe for non-target organisms and the environment (Boemare et al., 1996; Ehlers 2003). EPNs can also be mass-produced in liquid culture (Lunau et al., 1993; Ehlers et al., 1998; Strauch \& Ehlers, 1998; Ehlers, 2001) for widespread commercial use. Furthermore, Infective Juveniles (IJs) can resist shear stress; standard pesticide sprayers or irrigation systems can be used for application (Georgis, 1990; Wright et al., 2005). The use of EPNs has been growing worldwide, but some limiting factors prevent the use of EPNs in certain regions. These initial factors include heat, desiccation, persistence, and effectiveness (Strauch et al., 2000). Heat is one of the major stress factors for EPNs in soil conditions. Generally, the optimal temperature for EPNs is approximately $25^{\circ} \mathrm{C}$; however, some strains can handle temperatures up to $40{ }^{\circ} \mathrm{C}$ without any adaptation, or genetic selection (Koppenhöfer, 2000; Ulu \& Susurluk, 2014). Several studies have achieved improvement in these characteristics with hybridization (Mukuka et al., 2010a). For a commercial EPN strain, higher heat tolerance alone may not be enough. EPNs must survive several environmental conditions and have high effectiveness in order to be a feasible commercial product. Therefore, several characteristics and how these traits are related must be assessed to obtain a superior EPN strain.

The aim of the present study was to determine and compare the effectiveness of 4 strains from the following high-temperature-adapted EPN species: Heterorhabditis bacteriophora, Steinernema feltiae and S. carpocapsae. Thus, relationship between heat tolerance 
and virulence can be examined and data for further hybridization and genetic selection studies can be provided.

\section{Material and Methods}

\section{Nematode strains}

The following nematode strains used in the study were isolated from cities in Turkey: Heterorhabditis bacteriophora (H.b. 17) isolated in Kırklareli, H. bacteriophora (H.b. 1138) isolated in Antalya (H.b. 1138), Steinernema feltiae (TUR-S3) isolated in Ankara and S. carpocapsae (TUR-S4) isolated in Bursa. All strains were identified using a molecular technique (PCR-RFLP) (unpublished data). For soil sampling, ten soil samples $(500 \mathrm{~g})$ were taken from each area. Samples were merged in the laboratory to prevent labor loss. To isolate the nematodes, containers (with $100 \mathrm{gr}$ capacities) were filled with soil, and 3 last instar Galleria mellonella (Lepidoptera: Pyralidae) larvae were placed on the soil. The containers were incubated at $25^{\circ} \mathrm{C}$ for 3 days. Following the incubation, the dead larvae were transferred to a White trap. After two weeks after on a White trap, the nematodes emerged from the cadaver and were collected in a culture flask. The nematodes were then stored at $+4{ }^{\circ} \mathrm{C}$.
Table 1. Lethal temperatures of non-adapted and adapted populations of the strains $\left({ }^{\circ} \mathrm{C}\right)$

\begin{tabular}{lcccc}
\hline \multirow{2}{*}{ Strains } & \multicolumn{2}{c}{ Non-adapted populations } & \multicolumn{2}{c}{ Adapted populations } \\
\cline { 2 - 5 } & $\mathrm{LT}_{50}$ & $\mathrm{LT}_{90}$ & $\mathrm{LT}_{50}$ & $\mathrm{LT}_{90}$ \\
\hline H.b. 17 & 38.1 & 40.1 & 39 & 41.3 \\
H.b. 1138 & 37.4 & 40.4 & 37.7 & 40 \\
Tur S3 & 36.7 & 39 & 35.6 & 37.3 \\
Tur S4 & 35.5 & 38.7 & 35.7 & 38.2 \\
\hline
\end{tabular}

\section{Effectiveness}

High-temperature-adapted IJs from each of the 4 strains were inoculated on last instar $G$. mellonella larva at a dose of 1, 2, 3, 4 or $5 \mathrm{IJs} /$ larva. All doses were applied with a micro-injector. Inoculated larvae were put in each well of a 24-well plate. After the inoculation, plates were sealed with parafilm and incubated at $25^{\circ} \mathrm{C}$ for 4 days. The plates were opened at the end of the incubation period, and the dead larvae were dissected under a stereomicroscope to determine whether larvae were killed by the nematode.

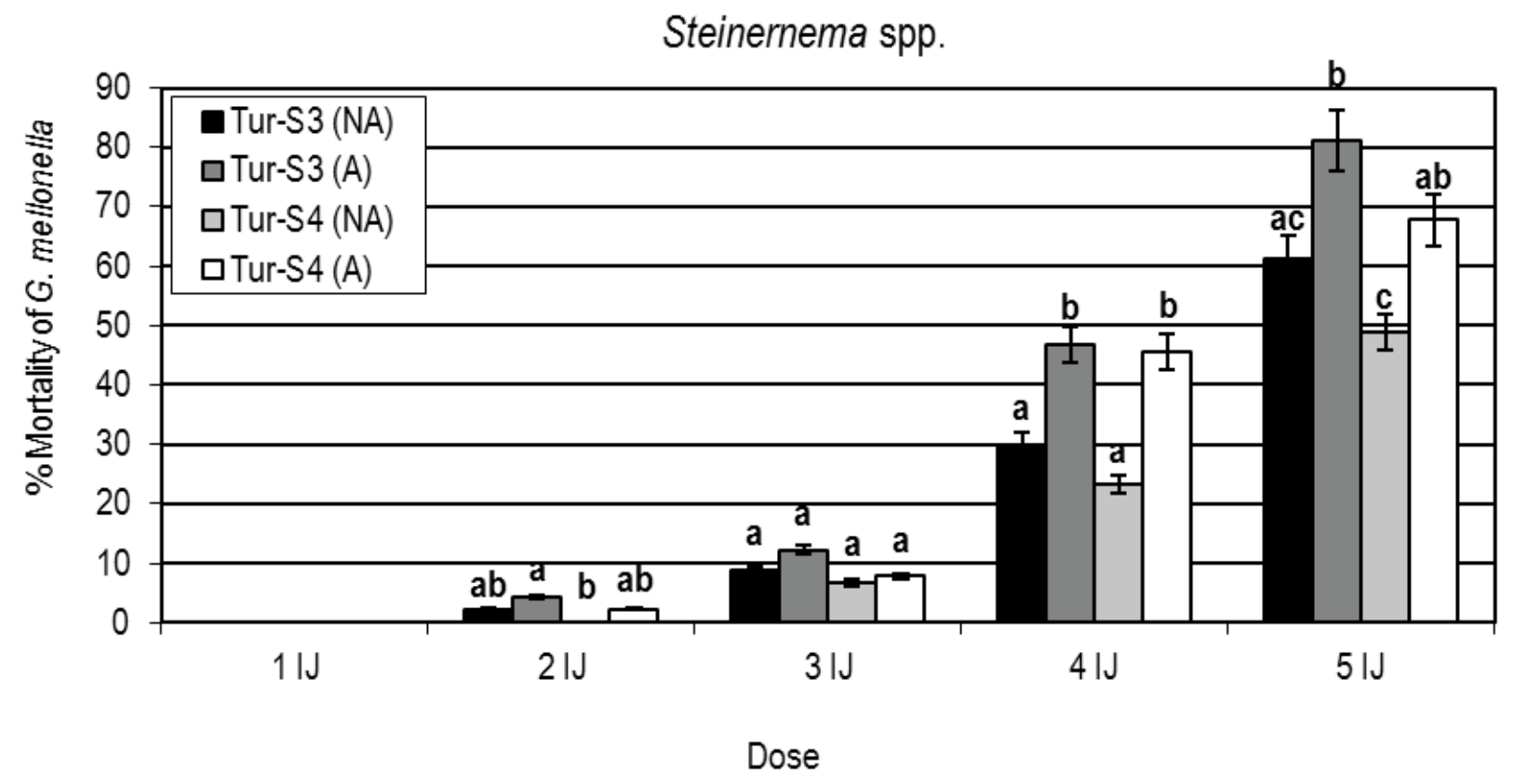

Fig. 1. Effectiveness to G. mellonella larvae comparison between heat adapted and non-heat adapted populations of Steinernema feltiae (TUR-S3) and Steinernema carpocapsae (TUR-S4) (NA = Non-Adapted, A = Adapted). For each dose, means followed by the same letter are not statistically different $(p>0.05)$

\section{Heat adaptation}

Isolated native strains were reproduced on G. mellonella as described by Kaya and Stock (1997). Heat adaptation experiments were conducted in 24-well tissue culture plates. For each well, 500 IJs of the desired strain were aliquoted with Ringer solution. Infective juveniles were exposed to $38^{\circ} \mathrm{C}$ for $2 \mathrm{~h}$ for heat adaptation before the effectiveness experiments were carried out. After the heat exposure, dead individuals were separated from living ones under a stereomicroscope. Live individuals were collected into a flask. The heat adaptation experiments were replicated 5 times.
After dissection, the mortality ratios of the strains were calculated. Effectiveness experiments were replicated 5 times using 30 larvae for each replicate.

\section{Statistical analysis}

For the heat adaptation experiments, $\mathrm{LT}_{50}$ and $\mathrm{LT}_{90}$ values were calculated by Probit analysis using BioStat 2010 software. To determine the difference between the effectiveness of the strains, ANOVA was performed at a $5 \%$ confidence level using JMP 7 software. Comparisons between the effectiveness of the strains 
were done using the Least Significant Differences (LSD) method at a $5 \%$ confidence level.

\section{Results}

\section{Heat adaptation}

Without adaptation, $\mathrm{Hb} 17$ was the most tolerant to heat of the four 4 strains according to both $\mathrm{LT}_{50}$ and $\mathrm{LT}_{90}$; Tur $\mathrm{S} 4$ was the least tolerant strain (Table 1). With adaptation, all strains developed some tolerance to heat (Table 2). Hb 17 was still the most tolerant

Table 2. Statistical analysis of the effectiveness of non-adapted and adapted populations of used species

\begin{tabular}{lcc}
\hline Dose & H.b. 17 and H.b. 1138 & Tur S3 and Tur S4 \\
\hline 1 IJ & - & $F=4.00 ; d f=3,8 ; P=0.519$ \\
2 IJ & - & $F=7.33 ; d f=3,8 ; P=0.011^{*}$ \\
3 IJ & $F=0.56 ; d f=3,8 ; P=0.658$ & $F=0.78 ; d f=3,8 ; P=0.538$ \\
4 IJ & $F=2.11 ; d f=3,8 ; P=0.177$ & $F=7.87 ; d f=3,8 ; P=0.009^{*}$ \\
5 IJ & $F=0.95 ; d f=3,8 ; P=0.960$ & $F=10.09 ; d f=3,8 ; P=0.043^{*}$
\end{tabular}

* Statistically significant at $p<0.05$ no significant differences between the adapted and non-adapted IJs were observed (Fig. 2) (Table 2).

\section{Discussion}

Entomopathogenic nematodes (EPNs) are one of the best biological alternatives to chemical agents against soil-dwelling insect pests (Ehlers, 2001). More than 80 species of EPNs have been identified; more than 11 species have been commercialized (Kaya \& Koppenhöfer, 1999). Shapiro-llan and Gaugler (2010) have listed the more the 25 commercial EPN producers worldwide. There are also several formulation types that contain different media and have different storage durations (Grewal, 2000).

Recent studies have generally focused on the effectiveness of EPNs on different insect pests and the improvement of several characteristics with genetic studies, such as selective breeding or hybridization (Mukuka et al., 2010a, 2010c; Nimkingrat et al., 2013; Susurluk et al., 2013; Ulu \& Susurluk, 2014). At present, one of the major obstacles in EPN commercialization is the high application cost due to short shelf life and high production expenses. Developing a superior EPN strain can be an alternative way to reduce application costs indirectly.

Anbesse et al. (2013) tried to improve the beneficial traits of entomopathogenic nematodes with genetic selection. Prior to these

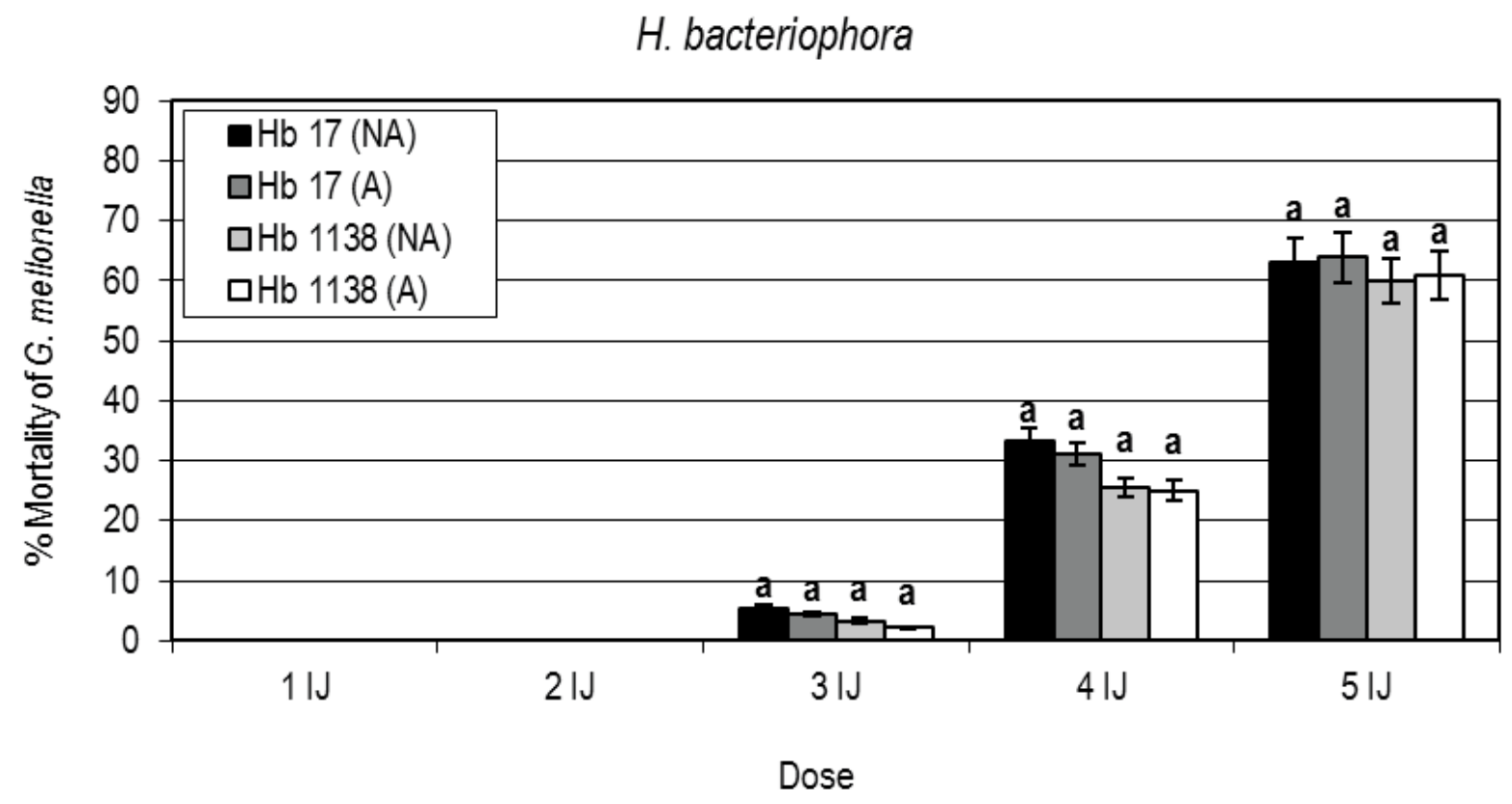

Fig. 2. Effectiveness to $G$. mellonella larvae comparison between heat adapted and non-heat adapted populations of $H$. bacteriophora (NA = Non-Adapted, $A=$ Adapted). For each dose, means followed by the same letter are not statistically different $(p>0.05)$

strain to heat after heat adaptation. The mortality of the larvae of G. mellonella increased with the dose, and significant differences were found between the adapted and non-adapted strains of Steinernema feltiae (TUR-S3) and S. carpocapsae (TUR-S4). At doses of 2, 4 and $5 \mathrm{IJ} /$ larva the heat-adapted populations of the Steinernema species were more effective than the non-adapted populations (Fig. 1). For the Heterorhabditis bacteriophora strains, trials, EPN cultures were reared in vivo and in vitro for 7 cycles with exposure to heat. Afterward, the EPNs reproduced in vivo and in vitro for 15 cycles without any selection pressure. In order to determine the effectiveness of the EPN cultures, $1200 \mathrm{lJ}$ from each culture were applied to the center of glass petri dishes in 1 $\mathrm{ml}$ of water, and 40 mealworms (Tenebrio molitor) were added. The results of this study indicated that the mean mortality of the 
commercial EN01 strain (70\%) and different subcultures of the D4 strain (in vivo and in vitro cultures reared under selection pressure) (> $70 \%$ ) were significantly greater than the untreated controls. It was also found that the mortality rate of the culture reared in vivo for 15 cycles without selection pressure was not significantly different from untreated cultures. These findings are in accordance with the present study, which its results showed that the heat treated populations of Steinernema spp. had greater mortality than untreated populations.

A similar study was performed by Mukuka et al. (2010b). The aim of this study was to determine the effect of temperature and desiccation stress on the infectivity of hybrid strains. Strains were exposed to $40{ }^{\circ} \mathrm{C}$ for heat tolerance and $0.85 \mathrm{a}_{\mathrm{w}}$ for desiccation tolerance prior to inoculation on $\mathrm{G}$. mellonella at a dose of $5 \mathrm{IJ} \mathrm{per}$ larva. The results showed that exposure to desiccation significantly reduced the mortality of the hybrids (below $25 \%$ ). Untreated hybrids had a mean infectivity of approximately $54 \%$. In contrast, the heat-tolerant hybrids had higher infectivity, with a mean mortality of $75.4 \%$. The effect of low temperature on infectivity was also investigated; however, no relation was found between these traits. Even though the populations that exposured to desiccation had lower mortality; the populations that exposured to heat had greater effectiveness than untreated populations, which obtained similarly as in the present study.

Another study by Mukuka et al. (2010c) analyzed the fitness of heat- and desiccation-tolerant hybrid strains of the species Heterorhabditis bacteriophora. The virulence of the hybrids was calculated by counting the dead-alive G. mellonella larvae that were inoculated with $2 \mathrm{IJs}$ each of a hybrid strain. As in the present study, tests were performed in 24 well-plates with sterile sand (with water at $10 \% \mathrm{w} / \mathrm{w}$ ). It was found that virulence in the two heat-tolerant hybrid strains $\mathrm{HH} 1(75.7 \%)$ and $\mathrm{HA} 1$ (71.3 \%) were significantly greater than the commercial hybrid strain EN01 (51.5\%), which showed the lowest heat tolerance. Their results are not similar with the present study, which treated and untreated populations of $H$. bacteriophora had no significant differences in virulence. However, the present results for Steinernema spp. in the study are in harmony with their findings.

This study plays a key role in the use of improved EPNs by hybridization in biological control systems. The results of the present study are expected to provide important support for sustainable biocontrol experiments using EPNs. However, further studies should investigate beneficial traits, such as heat, desiccation, and effectiveness.

\section{Acknowledgements}

This project was financially supported by the Scientific and Technological Research Council of Turkey (TÜBITAK) (Project Number: TOVAG 1100161). We would also like to thank e-nema $\mathrm{GmbH}$ for technical support and Prof. Dr. Ralf-Udo Ehlers for his practical insights.

\section{References}

Anbesse, S., Sumaya, N.H., Dörfler, A.V., Strauch, O., Ehlers, R.U. (2013): Stabilisation of heat tolerance traits in Heterorhabditis bac- teriophora through selective breeding and creation of inbred lines in liquid culture. BioControl 58, 85 - 93. DOI: 10.1007/s10526012-9467-X

Boemare, N.E., Laumond C., Mauleon, H. (1996): The entomopathogenic nematode-bacterium complex: Biology, life cycle and vertebrate safety. Biocontrol Sci. Technol., 6: 333 - 346. DOI: 10.1080/09583159631316

Ehlers, R.U., Lunau, S., Krasomil-Osterfeld, K., Osterfeld, K.H. (1998): Liquid culture of the entomopathogenic nematode-bacterium complex Heterorhabditis megidis / Photorhabdus luminescens. BioControl, 43: 77 - 86. DOI: 10.1023/A:1009965922794

EHLERS, R.U., (2003): Biocontrol nematodes. In: HokKANEN, H.M.T., HAJEK, A.J. (Eds) Environmental Impacts of Microbial Insecticides. Dordrecht:, Kluwer Academic Publishers, pp. 177 - 220

EHLERS, R.U., (2001): Mass production of entomopathogenic nematodes for plant protection. Appl. Microbiol. Biot., 56: 623 - 633.

GEORGIS, R., (1990): Formulation and application technology. In: Gaugler, R., KayA, H.K. (Eds) Entomopathogenic nematodes in biological control. Boca Raton, Florida: CRC Press, pp. 173 - 191 GREWAL, P.S. (2000): Enhanced ambient storage stability of an entomopathogenic nematode through anhydrobiosis. Pest Manag. Sci., 56: 401 - 406. DOI: 10.1002/(SICI)15264998(200005)56:5<401::AID-PS137>3.0.CO;2-4

KaYA H.K., KoppenhöFER, A.M. (1999): Biology and Ecology of Insecticidal Nematodes. In Workshop Proceedings: Poavarapu, S., (Ed) Optimal Use of Insecticidal Nematodes in Pest Management. Rutgers University, pp. 1 - 8

KaYA, H.K., Stock, S.P. (1997): Techniques in insect nematology. In: LACEY, L.A. (Ed) Techniques in insect pathology. London, UK: Academic Press, pp. 281 - 324

KoppenhöfER, A. (2000): Nematodes. In: LACEY, L.A, KayA H.K. (Ed) Field manual of techniques in invertebrate pathology. Dordrecht: Kluwer Academic Press, pp. 283 - 301

Lunau, S., Stoessel, S., Schmidt-Peisker, A.J., Ehlers, R.U. (1993): Establishment of monoxenic inocula for scaling up in vitro cultures of the entomopathogenic nematodes Steinernema spp. and Heterorhabditis spp. Nematologica, 39: 385 - 399. DOI: 10.1163/187529293X00330

Mukuka, J., Strauch, O., Al Zainab, M.H., Ehlers, R.U., (2010b): Effect of temperature and desiccation stress on infectivity of stress tolerant hybrid strains of Heterorhabditis bacteriophora. Russ. J. Nematol., 18: 111 - 116

Mukuka, J., Strauch, O., Hoppe, C., Ehlers, R.U. (2010c): Fitness of heat and desiccation tolerant hybrid strains of Heterorhabditis bacteriophora (Rhabditidomorpha: Heterorhabditidae). J. Pest Sci., 83: 281 - 287. DOI: 10.1007/s10340-010-0296-3

Mukuka, J., Strauch, O., Hoppe, C., Ehlers, R.U. (2010a): Improvement of heat and desiccation tolerance in Heterorhabditis bacteriophora through cross-breeding of tolerant strains and successive genetic selection. BioControl, 55: 511 - 521. DOI: 10.1007/ s10526-010-9271-4

Nimkingrat, P., Khanam, S., Strauch, O., Ehlers, R.U. (2013): Hybridisation and selective breeding for improvement of low temperature activity of the entomopathogenic nematode Steinernema feltiae. BioControl, 58: 417 - 426. DOI: 10.1007/s10526-012-9497-4 Shapiro-Ilan, D.I., Gaugler, R. (2010): Nematodes: Rhabditida: 
Steinernematidae \& Heterorhabditidae In: SHELTON, A. (Ed) Biological control: A Guide to natural enemies in North America. Cornell University, College of Agriculture and Life Sciences (http://www. biocontrol.entomology.cornell.edu)

Strauch, O., Ehlers, R.U. (1998): Food signal production of Photorhabdus luminescens inducing the recovery of entomopathogenic nematodes Heterorhabditis spp. in liquid culture. Appl. Microbiol. Biotechn., 50: 369 - 374. DOI: 10.1007/s002530051306

Strauch, O., Niemann, I., Neumann, A., Schmidt, A.J., Peters, A., EHLERS, R.U. (2000): Storage and formulation of the entomopathogenic nematodes Heterorhabditis indica and $H$. bacteriophora. BioControl, 45: 483 - 500. DOI: 10.1023/A:1026528727365
Susurluk, I.A., Ulu, T.C., Kongu, Y. (2013): Tolerances of hybridized entomopathogenic nematode Heterorhabditis bacteriophora (Rhabditida: Heterorhabditidae) strains to heat and desiccation. Turkish J. Entomol., 37: 221 - 228

ULu, T.C., Susurluk, I.A. (2014): Heat and desiccation tolerances of Heterorhabditis bacteriophora strains and relationships between their tolerances and some bioecological characteristics. Inv. Surv. J., 11: 4 - 10

Wright, D.J., Peters, A., Schroer, S., Fife, J.P. (2005): Application technology. In: Grewal, P.S., Ehlers, R.U., Shapiro-Ilan, D.I. (Eds) Nematodes as biological control agents. New York: CABI Publishing, pp. $91-106$ 